Research Article

\title{
"Snap-through" Buckling of Laminated Cylindrical Shells at Finite Rotations
}

\author{
M.N. Rao ${ }^{\dot{A}^{*}}$ and R. Schmidt ${ }^{\dot{A}}$ \\ ${ }^{\grave{A}}$ Institute of General Mechanics, RWTH Aachen University, Templergraben 64, Germany, D-52062
}

Accepted 10 January 2014, Available online 01 February 2014, Special Issue-2, (February 2014)

\begin{abstract}
This paper presents a finite element simulation and buckling analysis of layered composite structures. The shell element is based on the Reissner-Mindlin first-order shear deformation theory and the finite element method account for full geometric nonlinearity imposing large deformations and rotations. Finite rotations are treated by Rodrigues parameterization. The combinations of enhanced assumed strain (EAS) in the membrane strains and assumed natural strains in the shear strains are implemented to improve the shell element behavior. Stability analysis of anisotropic short open cylinder, layered cylindrical shells with different lamina sequenceand a hinged roof structure are presented including snap-through and snap-back problems. The present simulations are compared with those obtained by finite element analyses based on first-order transverse shear deformation moderate or large rotation or refined von Kármántype theories in earlier literature.
\end{abstract}

Keywords: Geometric Nonlinearity, Finite Rotations, Composite Plates and Shells, FE-analysis, Snap-through Problems

\section{Introduction}

Due to their high specific modulus compared to conventional materials, composite materials are increasingly being used in various structural applications. These layered composite thin-walled structures exhibits quite unique response even under simple loading and geometric conditions, due to the low natural damping. The challenge in this field of engineering exists to improve the static as well as dynamic behavior of structures.

The finite element analysis of shell structures focusing on computational aspects can be traced back to, (Ahmad,et al, 1970) presented degenerated 8-node shell element from 16-node three-dimensional continuum elements. (Chang and Sawamiphakdi, 1981) analyzed large deformations of a thin-walled cylinder under internal pressure using degenerated approach and updated Lagrangian formulation. (Jun and Hong, 1988) used very similar approach to analyze buckling behavior of laminated cylindrical shells. (Kanok-Nukulchai, et al, 1988) developed finite element formulations of two-degenerated thin-walled elements accounting wrapping restraint.In a degenerated shell element, nodes are placed at the midsurface of the element and assumptions of shell elements are imposed. (Saigal, et al, 1986) simulated nonlinear finite element (FE) analysis of imperfect laminated shells using Kirchhoff-Love model. (Schmidt and Reddy, 1988), (Palmerio, Reddy and Schmidt, 1990) used first-order shear deformation model (FOSD) to simulate the layered composite structures with in the moderate rotations and extended to large rotation analysis by (Kreja, Schmidt and

*Corresponding author: M.N. Rao

DOI: http://dx.doi.org/10.14741/ijcet/spl.2.2014.125
Reddy, 1997). (Brank, et al, 1995) developed layer-wise shell theory with the description of rotations using shell director. (Reddy, 1990) analyzed FE-simulation of moderately thick shells with in the von Kármán-type nonlinearityusing third-order shear deformation hypothesis (TOSD). Very less literature can be found on the geometrically nonlinear problems dealing with large rotations (e.g. Başar, et al, 1993, Masud,et al,2000, Kreja and Schmidt, 2006, Arciniegaand Reddy, 2007).

To analyze the thin walled and shell structures, in addition, solid-shell elements with eight nodes are being widely used. (Dommisy, et al, 1995) and (Sze,et al, 1997) are the first authors to investigate a solid-shell approach for the analysis of plates and shells.Solid-shell elements have only translational degrees of freedom in the nodes located at the top and bottom surfaces, which alleviates the difficulties associated with complex shell formulations with nodal rotations.

For instance, shear locking effects are usual in plate and shell finite elements based on Reissner-Mindlin or FOSD formulations, as thickness values tend to zero. The efforts by many investigators have been directed at overcoming the transverse shear locking problem. Solutions originally proposed to alleviate locking are reduced integration or selective integration. Another type of method which is adopted in the present element is the assumed natural strain (ANS) and enhanced assumed strain (EAS) method. The early use of ANS method can be traced to (Hughges and Tezduyar, 1981), (Bathe and Dvorkin, 1985). A systematic development of a class of assumed strain methods is presented by (Simo and Rifai, 1990). The EAS method was studied by (Andelfinger and Ramm, 1993) and (Fontes Valente,et al, 2005) to improve 
the behavior of the shell elements. (Kim,et al, 2007) developed 4-node co-rotational shell element with assumed natural strain and with enhanced strains to analyze the laminated composite structures. (Caseiro, et al, 2013) gave brief description and development of EAS three-dimensional finite elements for the alleviation of locking phenomena. (Pramin, et al, 2012) developed corotational 8-node degenerated element with assumed natural and enhanced assumed strains. (Cho,et al, 1998) proposed a solid-shell element model based on the assumed strain formulation for buckling and post-buckling analysis of shell structures. (Kinkel,et al, 2007) developed solid-shell element with assumed natural strain and enhanced assumed strains by parameterizing the finite rotations with Rodrigues approach.

The present paper deals with geometrically nonlinear static analysis of composite plate and 'snapthrough'buckling analysis of composite laminated cylindrical shell structures in the framework of the firstorder transverse shear deformation theory (FOSD). The theory and the finite element method account for full geometrical nonlinearity, thus they account for arbitrary, finite rotations. The finite rotation theory (FRT) finite element formulation developed by (Lentzen, 2009) and extendedto stabilityanalysis in (Schmidt, et al, 2013) is applied. Here we deal with geometrically nonlinear static and stability problems of composite laminated structures. The Riks-Wempner-Ramm arc-length control method is used to trace equilibrium paths in the pre- and postbuckling range of deformation.

We consider standard benchmark problems of composite laminated plate and shell structures. In particular, we compare the results with those that have been obtained in our earlier paper (Kreja and Schmidt, 2006) where comparative finite element analysis has been performed using a variety of geometrically nonlinear shell theories.

\section{Finite Rotation Shell Theory and Finite Element Method}

Concerning the underlying theory and finite element method, the present paper is based on the FOSD shell theory for small strains and finite rotations of (Habip, 1965) and the FE implementation of (Gruttmann and Wagner, 2006) and (Lentzen, 2009).

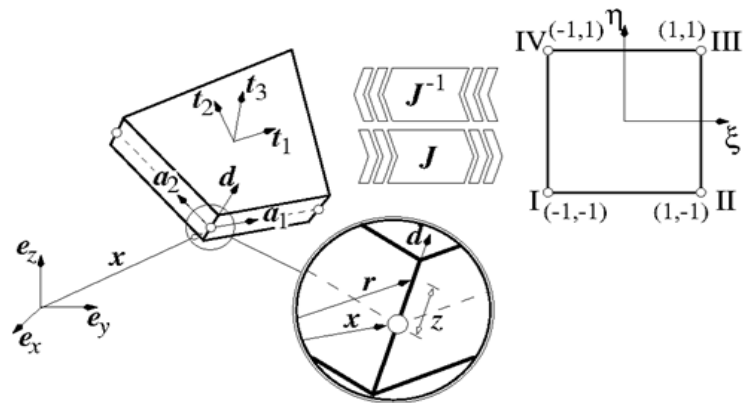

Fig.1 Definition of the coordinate systems in the FRTelement

Figure 1 shows the four-node element with the global $\boldsymbol{e}_{i}$, the local $\boldsymbol{a}_{\alpha}$, the natural $\xi-\eta$ and the orthonormal material $\boldsymbol{t}_{i}$ coordinate systems. The position vector to the reference surface in the initial configuration at node $I$ is denoted as $\boldsymbol{x}_{\boldsymbol{I}}$. The Jacobian matrix $\boldsymbol{J}$ can be used to relate the local coordinate system to the natural coordinate system. The position vector $r$ to an arbitrary point in shell space is described as

$r=\boldsymbol{x}(\xi, \eta)+z \boldsymbol{d}(\xi, \eta)$

where $z$ denotes the thickness coordinate ranging from the bottom to the top of the considered shell and $d$ is the shell director.

In the framework of FOSD or Reissner-Mindlin hypothesis, the Green strain-tensor components for fully nonlinear finite rotation strain-displacement relations are obtained as (see Habip (1965))

$\begin{array}{ll}\varepsilon_{\alpha \beta}=\stackrel{0}{\varepsilon}_{\alpha \beta}+\zeta \stackrel{1}{\varepsilon}_{\alpha \beta}+\zeta^{2} \stackrel{2}{\varepsilon}_{\alpha \beta} \\ \varepsilon_{\alpha 3}={\stackrel{0}{\varepsilon_{\alpha 3}}+\zeta \stackrel{1}{\varepsilon}_{\alpha 3}} & \varepsilon_{33}=0\end{array}$

Where

$$
\begin{aligned}
& \stackrel{0}{\varepsilon}_{\alpha \beta}=\frac{1}{2}\left(\overline{\boldsymbol{x}}_{, \alpha} \cdot \overline{\boldsymbol{x}}_{, \beta}-\boldsymbol{x}_{, \alpha} \cdot \boldsymbol{x}_{, \beta}\right) \\
& \stackrel{1}{\varepsilon} \alpha \beta_{\alpha}=\frac{1}{2}\left(\overline{\boldsymbol{x}}_{, \alpha} \cdot \overline{\boldsymbol{d}}_{, \beta}+\overline{\boldsymbol{x}}_{, \beta} \cdot \overline{\boldsymbol{d}}_{, \alpha}-\boldsymbol{x}_{, \alpha} \cdot \boldsymbol{d}_{, \beta}-\boldsymbol{x}_{, \beta} \cdot \boldsymbol{d}_{, \alpha}\right) \\
& \stackrel{2}{\varepsilon}_{\alpha \beta}=\frac{1}{2}\left(\overline{\boldsymbol{d}}_{, \alpha} \cdot \overline{\boldsymbol{d}}_{, \beta}-\boldsymbol{d}_{, \alpha} \cdot \boldsymbol{d}_{, \beta}\right) \\
& {\stackrel{0}{\varepsilon_{\alpha 3}}}=\frac{1}{2}\left(\overline{\boldsymbol{x}}_{, \alpha} \cdot \overline{\boldsymbol{d}}-\boldsymbol{x}_{, \alpha} \cdot \boldsymbol{d}\right) \\
& \stackrel{1}{\alpha 3}=\frac{1}{2}\left(\overline{\boldsymbol{d}}_{, \alpha} \cdot \overline{\boldsymbol{d}}-\boldsymbol{d}_{, \alpha} \cdot \boldsymbol{d}\right) .
\end{aligned}
$$

Here $\zeta$ denotes the thickness coordinate and an overbar indicates the deformed configuration. Due to thinness of the regarded plates and shells the components $\stackrel{2}{\varepsilon} \alpha \beta_{\alpha \beta}$ and $\varepsilon_{\alpha 3}$ are neglected.

There are no kinematical restrictions with respect to the range of rotations. The director is assumed to be inextensible and the rotations are parameterized with the Rodriguez formulation.In order to overcome various types of locking, the ANS procedure suggested by (Bathe and Dvorkin, 1985), (Hughes and Tezduyar, 1981) has been used.

\subsection{Variational formulation}

The finite element developed by (Lentzen, 2009) is based on the shell element of (Gruttmann and Wagner, 2006). In the present element a displacement based variational principle is chosen in order to reduce the number of internal variables. The variational formulation reads

$\int_{V}(\boldsymbol{S}: \delta \widehat{\boldsymbol{\varepsilon}}) d V=\int_{A}(\boldsymbol{T} \cdot \delta \boldsymbol{v}) d A+\int_{V}(\rho(\boldsymbol{b}-\ddot{\boldsymbol{v}})) \cdot \delta \boldsymbol{v} d V$

where $\boldsymbol{S}$ is the second Piola-Kirchhoff stress tensor, $\hat{\boldsymbol{\varepsilon}}$ is the strain field, $\delta \boldsymbol{v}$ is the virtual displacement vector, $\boldsymbol{T}$ is the traction vector on the boundary, $\rho$ is the mass density, $\boldsymbol{b}$ is the body force density and $\ddot{\boldsymbol{v}}$ is the acceleration vector.

The strain field is approximated by two fields such as Green-Lagrangian strain field $\varepsilon$ and enhanced strain field $\tilde{\varepsilon}$ as

$\widehat{\varepsilon}=\varepsilon+\tilde{\varepsilon}$ 
The enhanced strain field is approximated by the following relation

$\{\tilde{\varepsilon}\}=\frac{\left|J_{0}\right|}{|J|}\left[N_{E}\right]\left\{\alpha_{E}\right\}$

where $|\boldsymbol{J}|$ denotes the determinant of Jacobian matrix $\boldsymbol{J}, \boldsymbol{J}_{\mathbf{0}} \mid$ denotes the determinant of Jacobian matrix $\boldsymbol{J}_{\mathbf{0}}=\boldsymbol{J} \mid \xi=\eta=0$ at the element centroid in the natural coordinate system, $\left[\boldsymbol{N}_{\boldsymbol{E}}\right]$ is the interpolation function with enhancement levels, $\left\{\boldsymbol{\alpha}_{E}\right\}$ is the vector of the internal strain parameters corresponding to the enhanced strains.

Detailed description of level of enhancement, corresponding interpolation functions and following additional internal degrees of freedom authors referred to EAS approach in (Lentzen, 2009).

The variation of the Green-Lagrange strain tensor components are

$$
\begin{gathered}
\delta \varepsilon_{\alpha \beta}^{0}=\frac{1}{2}\left(\delta \overline{\boldsymbol{x}}_{, \alpha} \cdot \overline{\boldsymbol{x}}_{, \beta}+\overline{\boldsymbol{x}}_{, \alpha} \cdot \delta \overline{\boldsymbol{x}}_{, \beta}\right) \\
\delta \varepsilon_{\alpha \beta}^{1}=\frac{1}{2}\left(\delta \overline{\boldsymbol{x}}_{, \alpha} \cdot \overline{\boldsymbol{d}}_{, \beta}+\delta \overline{\boldsymbol{x}}_{, \beta} \cdot \overline{\boldsymbol{d}}_{, \alpha}+\overline{\boldsymbol{x}}_{, \alpha} \cdot \delta \overline{\boldsymbol{d}}_{, \beta}+\overline{\boldsymbol{x}}_{, \beta}\right. \\
\left.\delta \overline{\boldsymbol{d}}_{, \alpha}\right)
\end{gathered}
$$

$\delta \varepsilon_{\alpha 3}^{0}=\frac{1}{2}\left(\delta \overline{\boldsymbol{x}}_{, \alpha} \cdot \overline{\boldsymbol{d}}+\overline{\boldsymbol{x}}_{, \beta} \cdot \delta \bar{d}\right)$

With $\overline{\boldsymbol{x}}=\boldsymbol{x}+\boldsymbol{v}, \quad$ itfollows that $\delta \overline{\boldsymbol{x}}_{, \alpha}=\delta \boldsymbol{v}_{, \alpha} . \quad$ The variation of the director and its linearization can beobtained by

$\delta \overline{\boldsymbol{d}}=\boldsymbol{\delta} \boldsymbol{w} \times \overline{\boldsymbol{d}}$

where $\delta \boldsymbol{w}$ is the axial vector of rotation expressed in terms of virtual rotations as

$\delta \boldsymbol{w}=\boldsymbol{H} \delta \boldsymbol{\omega}$

and $\boldsymbol{H}$ is determined as

$\boldsymbol{H}=\boldsymbol{I}+c_{1} \boldsymbol{W}+c_{2} \boldsymbol{W}^{2}$

with $c_{1}=\frac{1-\cos \psi}{\psi^{2}}$ and $c_{2}=\frac{\psi-\cos \psi}{\psi^{3}}$

where $\boldsymbol{W}$ is the rotation tensor and $\psi$ is the rotation angle.

Here a total Lagrangian approach is adopted and since the kinematical relations between the strain and the displacements and the rotations is nonlinear, the second variation is

$$
\Delta \delta \Pi=\int_{V}(\Delta \boldsymbol{S}: \delta \widehat{\boldsymbol{\varepsilon}}+\boldsymbol{S}: \Delta \delta \widehat{\boldsymbol{\varepsilon}}) d V
$$

The variation of $\overline{\boldsymbol{d}}$ is highly nonlinear and the scalar multiplication of an arbitrary vector $\boldsymbol{k}$ with the linearization $\Delta \delta \overline{\boldsymbol{d}}$ is

$$
\boldsymbol{k} \cdot \Delta \delta \overline{\boldsymbol{d}}=\delta \boldsymbol{w} \boldsymbol{M}(\boldsymbol{k}) \Delta \boldsymbol{w}
$$

where

$$
\begin{gathered}
M(k)=\frac{1}{2}(\bar{d} \otimes \boldsymbol{k}+\boldsymbol{k} \otimes \overline{\boldsymbol{d}})+\frac{1}{2}(\boldsymbol{t} \otimes \boldsymbol{w}+\boldsymbol{w} \otimes \boldsymbol{t}) \\
+c_{10} I
\end{gathered}
$$

with

$\boldsymbol{t}=-c_{3} z+c_{11}(z \cdot w) w, z=\bar{d} \times \boldsymbol{k}$,

$c_{3}=\frac{\psi \sin \psi+2(\sin \psi-1)}{\psi^{2}(\cos \psi-1)}, c_{10}=\frac{\sin \psi-\psi}{2 \psi(\cos \psi-1)}(z \cdot w)-(\overline{\boldsymbol{d}} \cdot \boldsymbol{k})$,

$c_{11}=\frac{4(\cos \psi-1)+\psi^{2}+\psi \sin \psi}{2 \psi^{4}(\cos \psi-1)}$.

\subsection{System matrices and vectors}

After introducing the shape functions to interpolate mechanical quantities, the generalised displacements $\{q\}$ and integrated constitutive matrices $\left[H_{1}\right]$ for the elastic material, the following set of equations can be derived on element level from the variational formulations

$\left[\begin{array}{ll}{\left[K_{q q}\right]} & {\left[K_{q \alpha}\right]} \\ {\left[K_{\alpha q}\right]} & {\left[K_{\alpha \alpha}\right]}\end{array}\right]^{e}\left\{\begin{array}{c}\{\Delta q\} \\ \{\Delta \alpha\}\end{array}\right\}^{e}=\left\{\begin{array}{c}\left\{f_{q}^{q}\right\}-\left\{f_{q}^{i}\right\} \\ \left\{f_{\alpha}^{i}\right\}\end{array}\right\}^{e}$

where

$\left[K_{q q}\right]^{e}=\left[K^{\sigma}\right]^{e}+\int_{\Omega}\left[B_{q}\right]^{T}\left[H_{1}\right]\left[B_{q}\right] d \Omega$

$\left[K_{q \alpha}\right]^{e}=\left[K_{\alpha q}\right]^{e T}=\int_{\Omega}\left[B_{q}\right]^{T}\left[H_{1}\right]\left[N_{E}\right] d \Omega$

$\left[K_{\alpha \alpha}\right]^{e}=\int_{\Omega}{ }\left[N_{E}\right]^{T}\left[H_{1}\right]\left[N_{E}\right] d \Omega(10)$

The incremental stiffness matrix $\left[K_{q q}\right]$ is the only matrix containing the geometrical nonlinear stiffness $\left[K^{\sigma}\right]$ Further, $\left\{f_{q}^{q}\right\}$ is the vector containing the nodal forces and moments, $\left\{f_{q}^{i}\right\}$ is the vector containing imbalance forces, $\left\{f_{\alpha}^{i}\right\}$ is the vector containing the in balance forces for additional internal degrees of freedom.

\section{Numerical examples}

3.1 Asymmetric cross-ply laminated plate strip under uniformly distributed transverse load

Here, the considered example is an asymmetric cross-ply laminated plate with hinged edges as shown in Figure 2. The geometry is defined by $\mathrm{a}=9.0 \mathrm{in}, \mathrm{b}=1.5 \mathrm{in}, \mathrm{h}=0.04$ in and the material properties are taken as $E_{11}=2.0 \cdot 10^{7}$ psi, $E_{22}=1.4 \cdot 10^{6} \mathrm{psi}, v_{12}=0.3, G_{12}=G_{13}=G_{23}=0.7 \cdot 10^{6}$ psi.

The deflection at the center of the plate is simulated by the present FOSD FRT model. Due to the dual symmetry of the analyzed structure, a quarter of the plate is modeled. In Figure 3 the normalized transverse deflection is compared with the existing literature given by (LRT56-Kreja, 2013), (Kim, et al, 2007), (Kreja and Schmidt, 2006) (LRT5, MRT5) together with the reference solutions of(Reddy, 1990) and (Basar,et al, 1993).
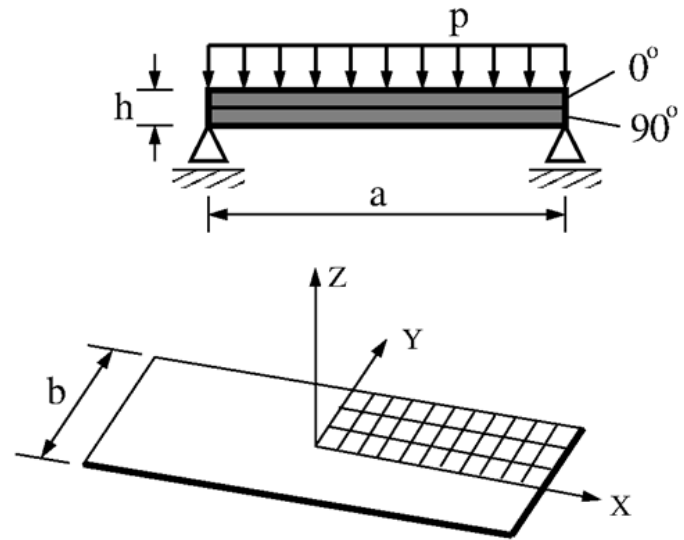

Fig.2 Two edges hinged composite laminated plate

The von Kármán-type model of (Reddy, 1990) and the (MRT5-Kreja and Schmidt, 2006) model are capable of dealing with deflections in the range of moderate rotations marked in Figure 3. (Basar,et al, 1993) solved this problem using a fully nonlinear formulation accounting for finite rotations along with higher order shear defamation theory. 


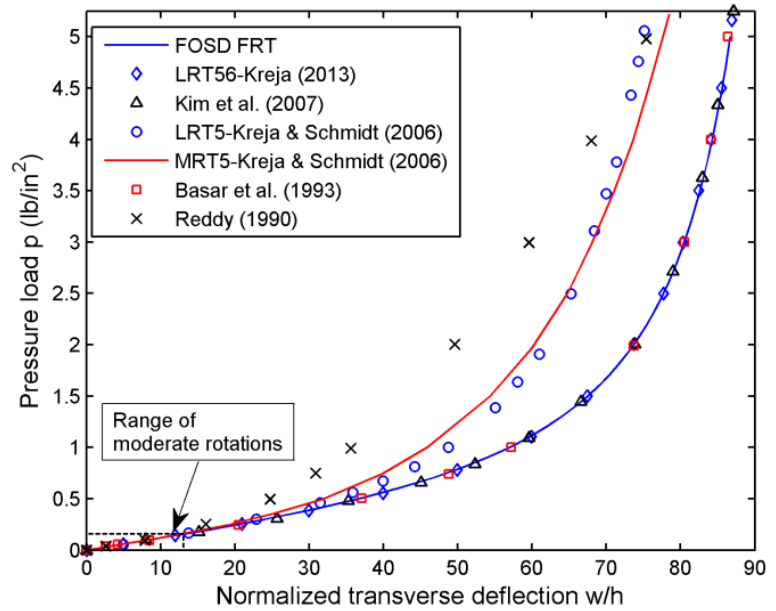

Fig.3 Normalized center deflection of the plate strip under pressure load

Our (FOSD FRT) results agree very well with those of (Basar, et al, 1993), (LRT56-Kreja, 2013) and (Kim,et al, 2007). On the other hand, the FOSD FRT solution differs from the other simplified nonlinear models LRT5 and MRT5.

\subsection{Stretching of an isotropic short cylinder}

The considered example is a well-known nonlinear benchmark problem of a short open cylindrical shell stretched by two opposite point loads as shown in Figure 4. This example constitutes a difficult test for finite element formulations, which induce large displacements and large rotations, following previous literature (Brank, Peric and Damjanic, 1995), (Sansourand Bednarczyk, 1995), (Chróścielewski, 1996), (Fontes Valente,et al, 2003),(Kreja and Schmidt, 2006), (Caseiro,et al, 2013) to name but a few. The geometric properties are the length $L$ $=5.175 \mathrm{in}$, the radius $R=4.953$ in and the thickness $h=$ $0.094 \mathrm{in}$. The material is isotropic with $E=10500 \mathrm{ksi}$ and $v$ $=0.3125$. No boundary conditions are applied to the free ends of the shell. Due to the symmetry, only one octant of the shell is modeled using $20 \times 32$ elements (20 along the length and 32 along the periphery).

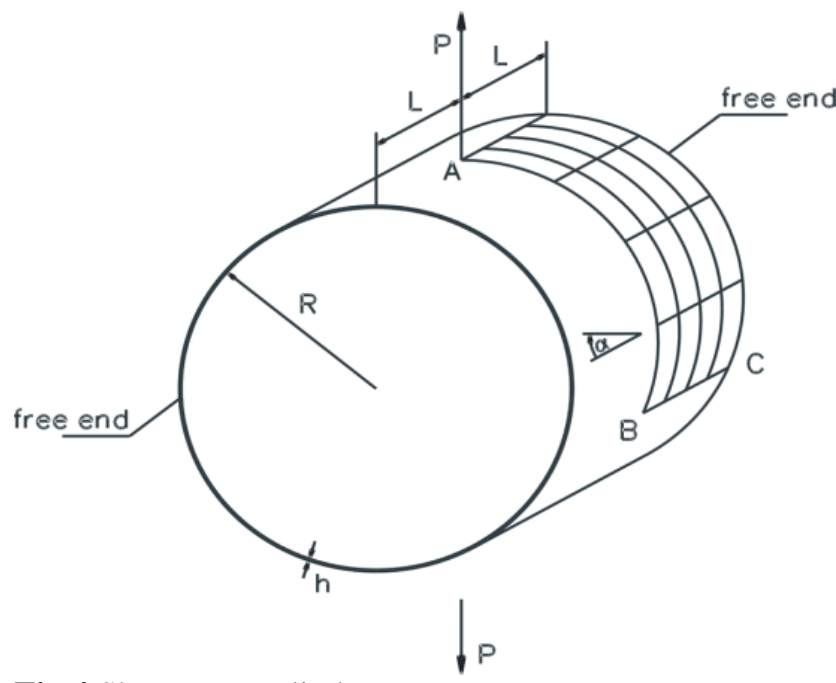

Fig.4 Short open cylinder

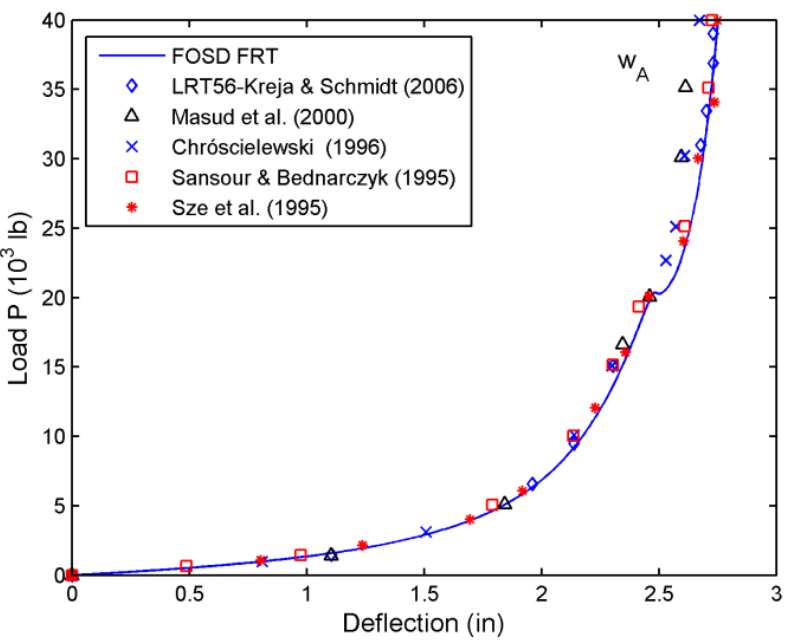

Fig. 5 Radial deflection at point A of isotropic cylinder

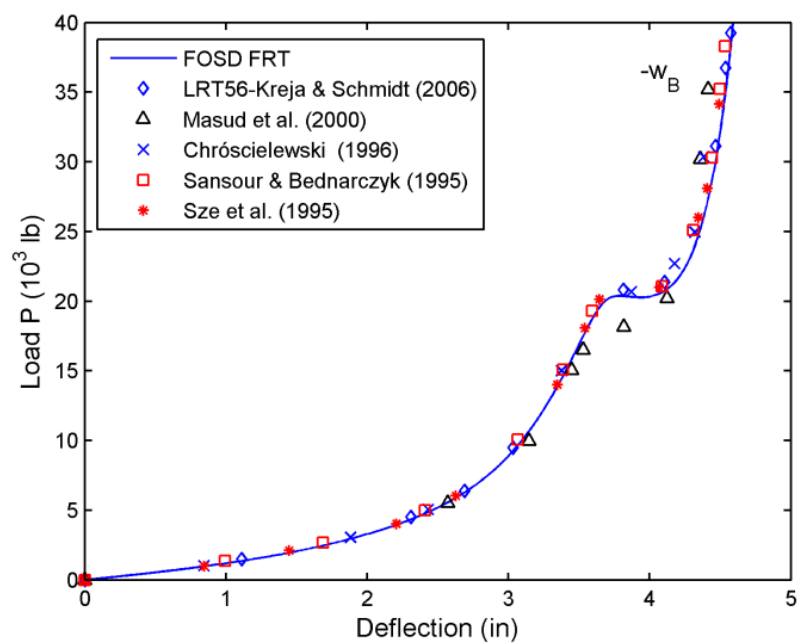

Fig. 6 Radial deflection at point B of isotropic cylinder

The load-deflection curves for the points $\mathrm{A}$ and $\mathrm{B}$ are presented in Figures 5 and 6 along with the reference solutions obtained in our earlier paper (Kreja and Schmidt, 2006) using a large rotation (LRT56) model, (SansourandBednarczyk. 1995), (Chróścielewski, 1996), (Masud,et al,2000)and (Sze,et al, 2004). The present results are also in a quite good agreement with those of (LRT56-Kreja and Schmidt,2006), (Sansour and Bednarczyk,1995), and (Chróścielewski,1996), while the solution of (Masud,et al, 2000) differs especially in the snap-through region, which is best visible in the loaddeflection curve for point $\mathrm{B}$.

\subsection{Stretching of composite laminated short cylinders}

In this, the same previous example is considered but with laminated composite layers. The geometry and boundary conditions are same as shown in Figure 4. The material properties of each layer are $E_{11}=30500 \mathrm{ksi}, E_{22}=10500$ ksi, $G_{12}=G_{13}=G_{23}=4000 \mathrm{ksi}$ and $v_{12}=0.3125$. Here two different cases of stacking sequences of the composite shell are analyzed. Due to the symmetry, only one octant of the shell is modeled using 20x32 elements (20 along the length and 32 along the periphery). 


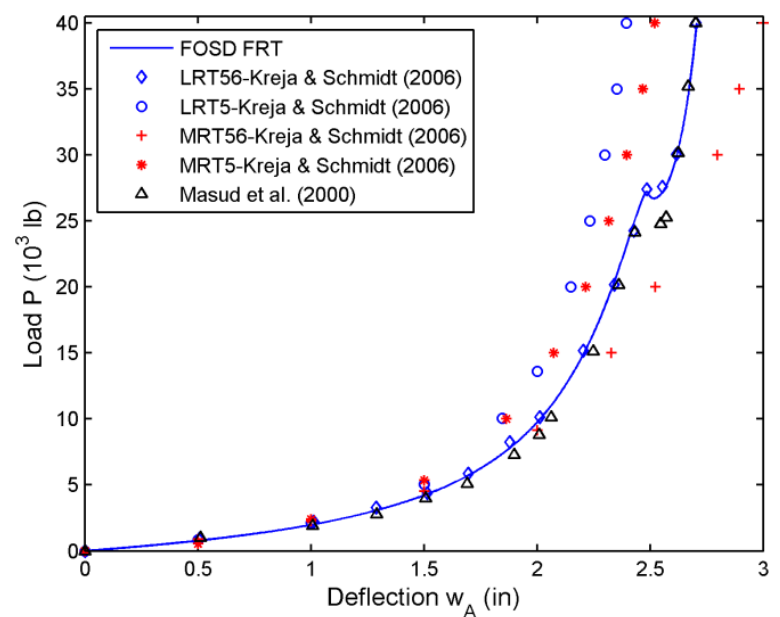

Fig. 7 Radial deflection at point $\mathrm{A}$ for $\left(0^{\circ} / 90^{\circ}\right)$ plies lay-up

In the first simulation, the composite shell with fiber orientations $\left(0^{\circ} / 90^{\circ}\right)$ is analyzed. In Figure 7 the radial displacement at point $\mathrm{A}$ obtained from the present $\mathrm{FE}$ formulation is compared with reference solutions of (Masud,et al, 2000) and (Kreja and Schmidt, 2006), who use two large (LRT5, LRT56), and two moderate rotation (MRT5, MRT56) theories. The results of the present FE model (FOSD FRT) are in excellent agreement with those of the LRT56 model. The result of (Masud,et al, 2000) differs in the snap-through region, while the LRT5 model fails to predict the buckling phenomenon at all. As expected, the moderate rotation theories (see (Librescuand Schmidt, 1988), (Schmidt and Reddy, 1988), Palmerio, Reddy and Schmidt, 1990), (Kreja, Schmidt and Reddy, 1997)) yield good results only in the range of moderate rotations.

In Figure 8 the radial displacement at points $\mathrm{B}$ and $\mathrm{C}$ obtained from the present FE formulation is compared with reference solutions of (Masud,et al, 2000), (LRT56Kreja and Schmidt, 2006). The results of the present FE model (FOSD FRT) are in excellent agreement with those of the LRT56 model.

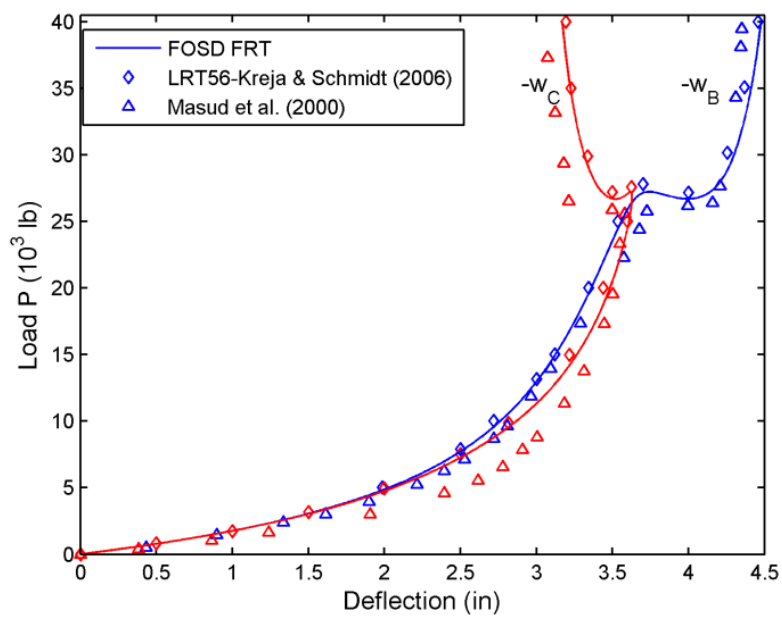

Fig. 8 Radial deflection at points $\mathrm{B}$ and $\mathrm{C}$ for $\left(0^{\circ} / 90^{\circ}\right)$ plies lay-up

In the second case, the numerical investigation is performed for the composite laminated short cylinder with lay-up $\left(90^{\circ} / 0^{\circ}\right)$. Figure 9 shows the radial displacements at points A, B and C, respectively. In Figure 9 the present simulations by FOSD FRT agree very well with those of (LRT56-Kreja and Schmidt, 2006).

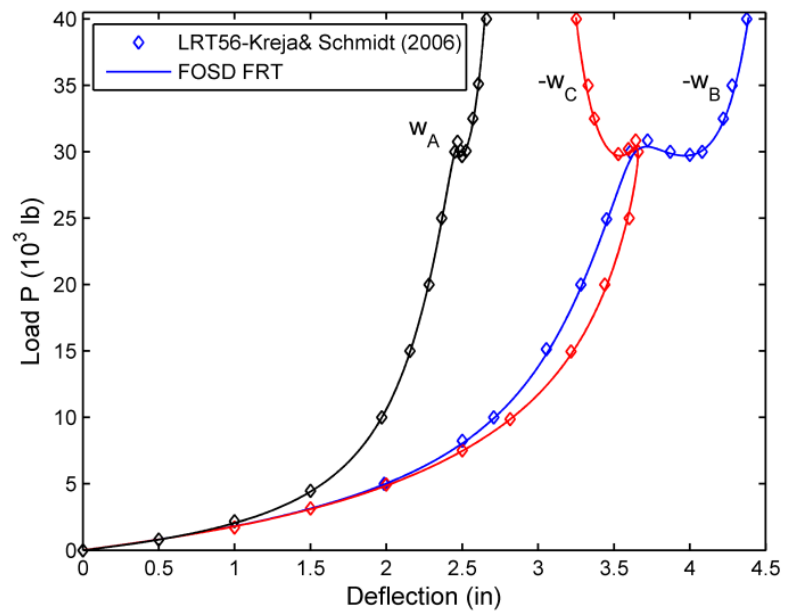

Fig. 9 Radial deflection at pointsA, B and C for $\left(90^{\circ} / 0^{\circ}\right)$ plies lay-up

\subsubsection{Influence of lamina sequence}

Figure 10 shows the radial displacements at points A, B and $\mathrm{C}$, respectively for different ply lay-ups $\left(90^{\circ} / 0^{\circ}\right)$ and $\left(0^{\circ} / 90^{\circ}\right)$. From Figure 10, it can be observed that the snapthrough load for stretching the composite laminated cylinder increases significantly, when the stacking sequence is changed from $\left(0^{\circ} / 90^{\circ}\right)$ to $\left(90^{\circ} / 0^{\circ}\right)$.

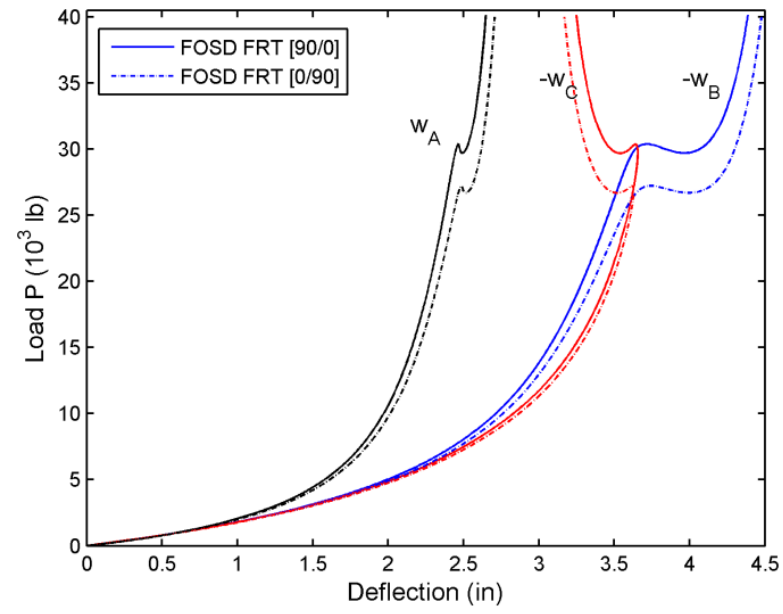

Fig. 10 Radial deflection at points A, B and C for $\left(90^{\circ} / 0^{\circ}\right)$ plies lay-up

In the third case, the numerical investigation is performed for the composite laminated short cylinders with lay-ups $\left(45^{\circ} / 45^{\circ}\right)$ and $\left(-45^{\circ} / 45^{\circ}\right)$. Figure 11 shows the radial displacements at points A, B and C, respectively.

From Figure 11, it is noticeable that the structure exhibits sudden buckling for lay-up $\left(-45^{\circ} / 45^{\circ}\right)$ and smooth snapthrough behavior for the lay-up $\left(45^{\circ} /-45^{\circ}\right)$. The snapthrough load for stretching the composite laminated cylinder increases significantly by $8 \%$, when the stacking sequence is changed from $\left(45^{\circ} /-45^{\circ}\right)$ and $\left(-45^{\circ} / 45^{\circ}\right)$. 


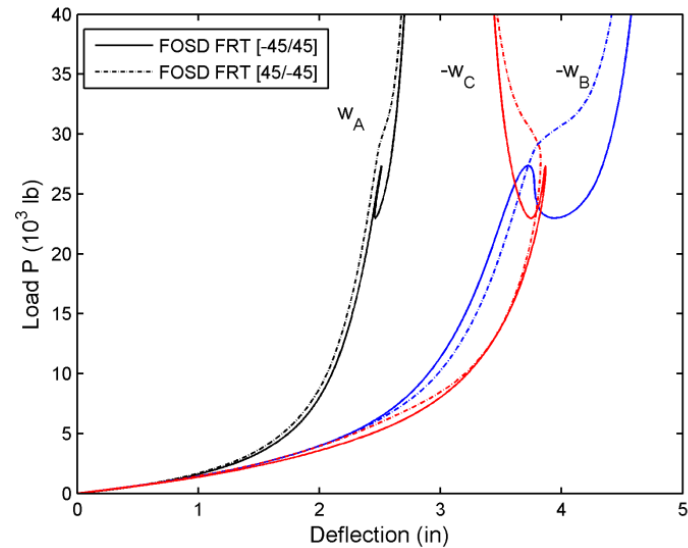

Fig. 11 Radial deflection at points A, B and C for $\left(90^{\circ} / 0^{\circ}\right)$ plies lay-up

\subsection{Unstable behavior of shallow-roof structure}

The considered example is a well-known nonlinear benchmark problem of a hinged cylindrical shell under a central point load. This example first considered by (Sabir and Lock, 1972), (Horrigome and Bergan, 1972) and latter it was extensively analyzed in literature (Ramm, 1982), (Cho,et al, 1998), (Crisfield, 1981), (Alves de Sousa,et al, 2006),(Pramin,et al, 2012) to name but a few. This example constitutes good test for finite element formulations, combining bending and membrane effects. The cylindrical shell is hinged along the longitudinal boundaries but unsupported along the curved edges. The geometry and boundary conditions for the model are presented in Figure 11. The material is taken as elastic with $E=3102.75 \mathrm{~N} / \mathrm{mm}^{2}$ and $v=0.3$.

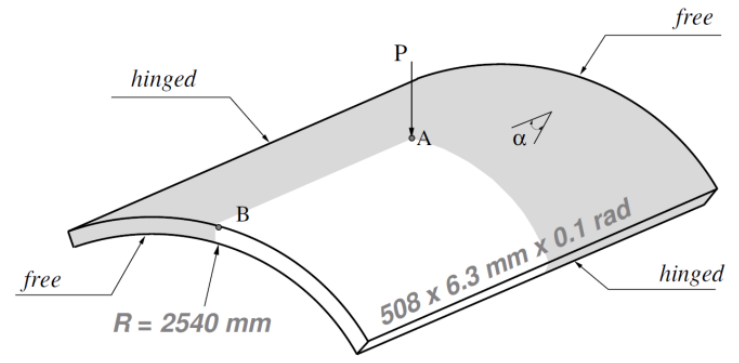

Fig. 12 Hinged cylindrical shell

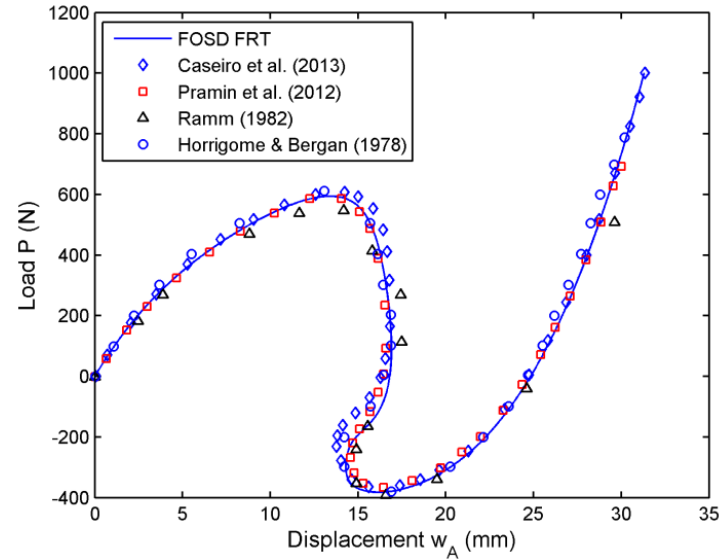

Fig. 13 Transverse deflection at point Aof hinged cylindrical shell

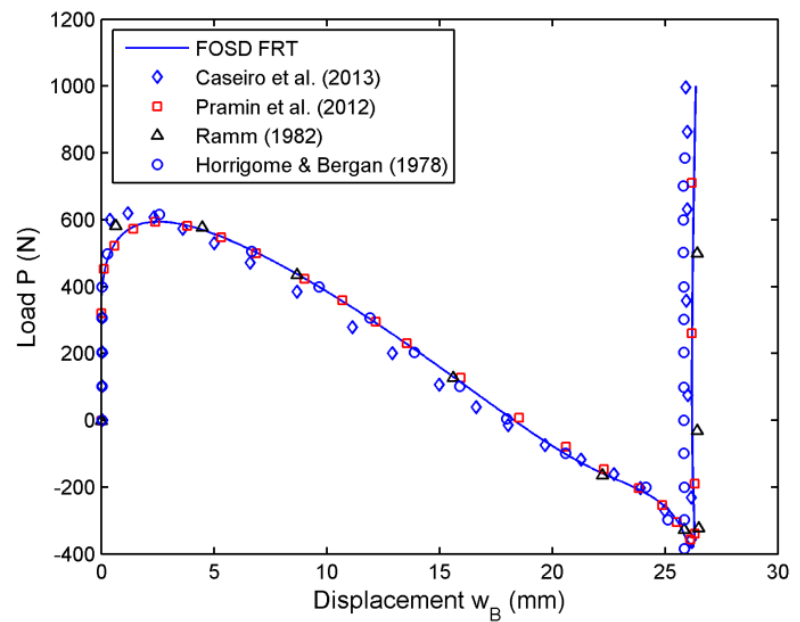

Fig. 14 Transverse deflection at pointB of hinged cylindrical shell

In this example, the snap-through and snap-back load displacements are analyzed. Due to the symmetry, only one quadrant of the shell is modeled using $8 \times 8$ elements.

The results of the $\mathrm{z}$ - displacement of points $\mathrm{A}$ and $\mathrm{B}$ are presented in Fig. 10 and Fig. 11, as well as solutions from the other existing literature given by (Horrigome and Bergan, 1978), (Ramm, 1982), (Pramin,et al, 2012), (Caseiro,et al, 2013) for comparison purposes. (Caseiro,et al, 2013) used 3D solid elements considering locking effects with subspace methodology. (Pramin,et al, 2012) used co-rotational 8-node solid element. Our FOSD FRT results agree very well with those of (Horrigomeand Bergan, 1978) and (Pramin, et al, 2012).

\section{Conclusions}

In this paper, nonlinear static analyses of a composite laminated plate strip subjected to uniform distributed load and stability analysis of an isotropic short cylinder and laminated cylindrical shellswith different stacking sequences and a hinged roof structure are simulated by FOSD FRT model. Additionally, influence of lamina sequence of pinched composite short cylinder also simulated by FOSD FRT model. The present simulations demonstrate the importance of the finite rotation models concerning a reliable consideration of nonlinearities.

\section{References}

T.J.R. Hughes, T.E. Tezduyar, (1981), Finite elements based upon Mindlin plate theory with reference to the four-node isoparametric element, International Journal for Numerical Methods in Engineering, vol.48, pp. 587-596

L.M. Habip, (1965), Thoery of elastic shells in reference state, Ing.Archi,34,pp. 228-237.

F. Gruttmann, W. Wagner, (2006), Structural analysis of composite laminates using a mixed hybrid shell element, Computational Mechanics, vol.37, pp. 479-497.

T.Y. Chang, K. Sawamiphakdi, (1981), Large deformation analysis of laminated shells by finite element method, Computers and Structures, vol.13, pp. 331-340.

K.J. Bathe, E, Dvorkin, (1985), A 4-node plate bending element based on Mindlin/Reissner theory and a mixed interpolation, International Journal for Numerical Methods in Engineering, vol.21, pp. 367-383 
R.A. Arciniega, J.N. Reddy, (2007), Tensor-based finite element formulation for geometrically nonlinear analysis of shell structures, Computer Methods in Applied Mechanics and Engineering, vol.196, pp. 1048-1073

Kreja, (2013), On geometrically non-linear FEA of laminated composite panels, Shell Structures: Theory and Applications, vol.3, pp. 33-42

Kreja, R. Schmidt, (2006), Large rotations in first-order shear deformation FE analysis of laminated shells, International Journal of Non-Linear Mechanics, vol.41, pp. 101-123

N. Pramin, S. Suthasupradit, K. Kim, (2012), A co-rotational 8-node degenerated thin-walled element with assumed natural strain and enhanced assumed strain, Finite Elements in Analysis and Design, vol.50, pp. 70-85

J.N. Reddy, (1990), A general nonlinear third-order theory of plates with moderate thickness. International Journal of Non-linear Mechanics, vol.25, pp. 677-686.

Y. Basar, Y. Ding, R. Schultz, (1993), Refined shear deformation models for composite laminates with finite rotations, International Journal of Solids and Structures, vol.30, pp. 2611-2638.

B. Brank, D. Peric, F.B. Damjanic, (1995), On implementation of a nonlinear four node shell finite element for thin multilayered elastic shells, Computational Mechanics, vol.16, pp. 341-359.

C. Sansour, H. Bednarczyk, (1995), The Cosserat surface as a shell model, theory and finite-element formulation, Computational Methods in Applied Mechanics and Engineering, vol.120, pp. 132.

J. Chróścielewski, (1996), Family of $\mathrm{C}^{0}$ finite elements in sixparameter non-linear theory of shells (in Polish), Habilitation Thesis, ZeszytyNaukowePolitechnikiGdanskiej, 540

R.A. Fontes Valente et al, (2003), On the use of an enhanced transverse shear strain shell element for problems involving large rotations, Computational Mechanics, vol.30, pp. 286-296.

J.F. Caseiro, R.J. Alves de Sousa, R.A.F. Valente, (2013), A systematic development of EAS three-dimensional finite elements for the alleviation of locking phenomena,Finite Elements in Analysis and Design, vol.73, pp. 30-41

K.Y. Sze, X.H. Liu, S.H. Lo, (2004), Popular benchmark problems for geometric nonlinear analysis of shells, Finite Elements in Analysis and Design, vol.40, pp. 1551-1569.

Masud, C.L. Tham, W.K. Liu, (2000), A stabilized 3-D co-rotational formulation for geometrically nonlinear analysis of multi-layered shells, Computational Mechanics, vol.26, pp. 1-12.

L. Librescu, R. Schmidt, (1988), Refined theories of elastic anisotropic shells accounting for small strains and moderate rotations, International Journal of Non-linear Mechanics, vol.23, pp. 217-229

R. Schmidt, J.N. Reddy, (1988), A refined small strain and moderate rotation theory of elastic anisotropic shells, ASME Journal of Applied Mechanics, vol.55, pp. 611-61

A.F. Palmerio, J.N. Reddy, R. Schmidt, (1990), On a moderate rotation theory of elastic anisotropic shells. Part 1: theory, Part 2: FE analysis. International Journal of Non-linear Mechanics, vol.25, pp. 687-714

Kreja, R. Schmidt, J.N. Reddy, (1997), Finite elements based on the first-order shear deformation moderate rotation shell theory with applications to the analysis of composite structures, International Journal of Non-linear Mechanics, vol.32, pp. 1123-1142

M. Crisfield, (1981), A fast incremental/iterative solution procedure that handles "snap-through", Computers and Structures, vol.13, pp. 55-62.

G. Horrigome, P.G. Bergan, (1978), Nonlinear analysis of free-form shells by flat finite elements, Computational Methods in Applied Mechanics and Engineering, vol.16, pp. 11-35.

A.B. Sabir, A.C. Lock, (1972), The application of finite elements to the large deflection geometrically nonlinear behavior of cylindrical shells, in: Variational methods in Engineering, University Press, Southampton, pp. 766-775.
E. Ramm, (1982), The Riks/Wempner approach-an extension of the displacement control method in nonlinear analyses, in: Recent Advances in Non-linear Computational Mechanics, Pineridge Press Limited, Swansea, UK, pp. 63-86

R.J. Alves de Sousa et al, (2006), A new one-point quadrature enhanced assumed strain EAS solid-shell element with multiple integration points along the thickness: Part II: nonlinear applications, International Journal for Numerical Methods in Engineering, vol.67, pp. 160-188.

C. Cho, H. C. Park, S.W. Lee, (1998), Stability analysis using a geometrically nonlinear assumed strain solid shell element model, Finite Elements in Analysis and Design, vol.29, pp. 121-135.

K.D. Kim, S.C. Han, S, Sathasupradit, (2007), Geometrically nonlinear analysis of laminated composite structures using a 4-node co-rotational shell element with enhanced strains, International Journal of Non-linear Mechanics, vol.42, pp. 864-881

S. Lentzen, (2009), Nonlinearly coupled thermopiezoelectric modelling and FE simulation of smart structures, $P h D$ Thesis RWTH Aachen University, Fortschritt-Berichte VDI 20: 419.

S. Saigal, R.K. Kapania, T.Y. Yang, (1986), Geometrically nonlinear finite element analysis of imperfect laminated shells,Journal of Composite Structures, vol.20, pp.197-214.

R. Schmidt, M.N. Rao, T.D. Vu, (2013), Modeling and nonlinear FE analyses of composite shells at finite rotations, Advances and Trends in Structural Engineering, Mechanics and Computation (Ed. A. Zingoni),Taylor \& Francis Group, London, UK, CRC Press Balkema, Leiden, The Netherlands, in print.

N. Stander, A. Matzenmiller, E. Ramm, (1989), An assessment of assumed strain methods in finite rotation shell analysis,Engineering Computations, vol.6, pp. 58-66.

C.T. Tsai, A.N. Palazotto, S.T. Dennis, (1991), Large rotation snapthrough buckling in laminated cylindrical panels, Finite Elements in Analysis and Design, vol.9, pp. 65-75.

J.N. Reddy, (1990),A general nonlinear third-order theory of plates with moderate thickness, International Journal of Non-Linear Mechanics, vol.25, pp.667-686.

S. Ahmad, B.M. Irons, O.C. Zienkiewicz, (1970), Analysis of thick and thin shell structures by curve finite elements, International Journal for Numerical Methods in Engineering, vol. 2, pp. 419451.

J.C. Simo, M.S. Rifai, (1990), A class of mixed assumed strain methods and the methods of incompatible modes, International Journal for Numerical Methods in Engineering, vol.29, pp. 15951638.

S.M. Jun, C.S. Hong, (1988), Buckling behavior of laminated composite cylindrical panels under axial compression, Computers and Structures, vol.29, pp.479-490.

W. Kanok-Nukulchai, M. Shivakumar, Degenerate element for combined flexural and torsional analysis of thin-walled structures, Journal of Structural Engineering, vol.114, pp. 657-674.

E. Domissy, S. Bouabdallah, J.L. Batoz, (1995), Formualtion and evaluation of a solid finite element model for the linear and nonlinear shell analysis, in:, 12eme CongrèsFrancais de Mécanique Strasbourg, France, (in French).

K.Y.Sze, S. Yi, M.H. Tay, (1997), An explicit hybrid-stabilized eighteen-node solid element for the shell analysis, International Journal for Numerical Methods in Engineering,vol.40, pp. 18391856.

U. Andelfinger, E. Ramm, (1993), EAS-elements for two dimensional, three dimensional, plate and shell structures and their equivalence to HR-elemetns, International Journal for Numerical Methods in Engineering, vol.36, pp. 1311-1337.

R.A. Fontes Valente et al, (2005), Enhanced transverse shear strain shell formulation applied to large elasto-plastic deformation problems, International Journal for Numerical Methods in Engineering, vol.62, pp. 1360-1398. 